\title{
Strangeness-changing response functions: an alternative approach to hypernuclear structure
}

\author{
H. Müller \\ Department of Physics, University of Colorado, Boulder, Colorado 80309 \\ J. Piekarewicz \\ Department of Physics and Supercomputer Computations Research Institute, \\ Florida State University, Tallahassee, Florida 32306-4130
}

(July 27, 2013)

\begin{abstract}
We study strangeness-changing response functions as an alternative description of hypernuclear structure. Hypernuclear states are treated in the same manner as any other conventional nuclear excitation that emerges from the response of the nuclear ground state to an external probe. The nuclear response is calculated using a random-phase approximation to an effective relativistic mean-field model that reproduces bulk properties of normal nuclei. The relevant meson-baryon vertices are constrained by imposing $\mathrm{SU}(3)$-flavor symmetry, while the residual hyperon-particle - nucleon-hole interaction is assumed to be mediated by the two lightest $S=-1$ mesons: the pseudoscalar kaon and its vector partner the $K^{*}(892)$. We use this model to analyze the spectra of ${ }_{Y}^{16} \mathrm{O}$ and ${ }_{Y}^{40} \mathrm{Ca}$ hypernuclei.
\end{abstract}

PACS number(s): 21.80.+a 


\section{INTRODUCTION}

Strangeness adds yet another — still largely unexplored — dimension to hadronic physics. For nucleons in free space, their strange-quark content has received considerable attention as a result of the measurement of the spin-dependent structure function of the proton by the European Muon Collaboration (EMC) [1]. Some analyses of the experiment suggest that a large portion of the spin of the proton is carried by strange quarks. In the opposite, highdensity, domain a copious production of strangeness is predicted as soon as the Fermi energy of the system becomes large enough for the addition of strange quarks to become energetically favorable [2]. Thus, one expects a substantial increase in the strangeness-per-baryon ratio with density. In addition to its obvious relevance to nuclear physics, high-density matter appeals to many other branches of physics, such as astrophysics, cosmology and particle physics. Indeed, the high-density environment found at the core of neutron stars is speculated to constitute a fruitful ground for the formation of exotic states of matter - such as a kaon condensate and strange-quark matter. Motivated by these fundamental issues a large number of experimental facilities are devoting valuable resources to elucidate them. However, before a clear picture of these exotic phenomena will emerge, it is necessary to understand phenomena at or near normal nuclear-matter density. The formation of hypernuclei appears to be an important first step. Moreover, because hyperon-nucleon scattering experiments are difficult to perform, hypernuclear physics appears to be an attractive alternative for testing — and for extending off the mass shell — models of the elementary nucleon-hyperon and hyperon-hyperon interaction. Indeed, a vast number of experiments have been devoted [3] to study the formation, the spectroscopy and the decay of hypernuclei.

To date, hypernuclei have been typically produced through strangeness-exchange reactions using hadronic probes - such as in $\left(K^{-}, \pi^{-}\right)$and $\left(\pi^{+}, K^{+}\right)$scattering - and decay through nonleptonic weak processes which involve the emission of nucleons and pions. These experiments have been analyzed from a variety of different theoretical perspectives. For example, microscopic meson-exchange models have been constructed which accurately reproduce the rich nucleon-nucleon and the more scarce hyperon-nucleon data [4,5]. Other approaches, more closely connected to the underlying symmetries of QCD, are based on effective field theories that include the baryon octet and several nonstrange and strange mesons [6 8]. With such models available, binding energies and single-particle spectra of hypernuclei have been computed in the context of nonrelativistic [9] and relativistic mean-field models [10]. The underlying assumption in the mean-field approach is that the dynamical aspects of the production and decay process can be ignored, so that the hypernucleus may be approximated as a core of nucleons with one strange baryon added to the system. If the hyperon has been produced from a closed-shell nucleus this approximation constitutes the simple particle-hole model. Yet one must take this simplified description with caution as the real picture is vastly more complicated. Indeed, it is widely recognized that the nuclear resonances populated through these reactions are often highly excited (particle-hole) states that may decay electromagnetically prior to their weak decay. Moreover, initial- and final-state interactions between the nucleus and the hadronic probes are strong and must be included. These aspects add considerable complexity to any theoretical description. Although the gross features of hypernuclear spectra may indeed be explained on the basis of a

simple particle-hole picture, the fine details require the incorporation of distortions as well 
as of few- and many-body correlation effects [11].

With the advent of continuous electron beam facilities, such as the Thomas Jefferson National Accelerator Facility (TJNAF), the formation of hypernuclear states through the photoproduction of charged kaons has become a reality [12]. The production of hypernuclei via hadronic or photo-nuclear reactions is a complicated process involving not only nucleons and hyperons but also several baryon resonances and strange mesons. In contrast to the hadronic production, however, the $\mathrm{K}^{+}$-photoproduction process is relatively insensitive to distortion effects. This represents an enormous theoretical simplification. Still, additional simplifications and approximations are often needed. For example, in order to keep the influence of the nuclear environment on a tractable level, most theoretical studies resort to the impulse approximation: the assumption that the elementary process suffers no modification as it is embedded in the nuclear medium. (Note that the free-space production operator could suffer considerably from model and parameter dependencies [13].) Further, if distortion effects can be neglected - an excellent approximation in the $K^{+}$-photoproduction case - then as in the case of electron scattering, the theoretical amplitude may be decomposed into a nuclear structure part and a production operator [13 [16]. The production operator contains the specific details of the reaction, including various meson-baryon vertices and form factors. It is obtained from the corresponding $T$-matrix describing the reaction on a single nucleon in free space. The nuclear structure part involves the response of the nuclear ground state in the form of several polarization insertions describing the propagation of a nucleon-hole and a $\Lambda\left(\Sigma^{0}\right)$-particle through the nuclear medium.

Using this point of view, we study hypernuclear spectra using a random-phase approximation (RPA) to a generalized relativistic mean-field model [17]. More specifically, baryonic matter is assumed to consist of strange as well as non-strange hadrons. At, or near, nuclearmatter saturation density - and for phenomenologically consistent hyperon-meson couplings - the presence of hyperons in the ground state is suppressed by their larger mass. Thus, at these densities one recovers the normal [strangeness-equal-zero $(S=0)$ ] nuclear ground state. Hypernuclear states, as well as any other nuclear excitation, then emerge from the consistent response of this mean-field ground state to a suitable external probe. That is, we propose to treat strangeness no different than any other intrinsic nuclear quantum number. Moreover, as the RPA is the consistent response of the mean-field ground state, all nuclear states - those with $S=0$ and $S \neq 0$ alike - are obtained by correlating the particle-hole pair via their residual interaction. The importance of many-body correlations beyond the simple particle-hole picture was realized soon after precise hypernuclear measurements were available [11]. For example, mixing of particle-hole states that couple to the same angular momentum is essential for predicting angular distributions in strangeness exchange reactions [18]. Furthermore, states with nucleon holes in the deepest bound nucleon shells are not recognized in the experiments because of their large widths, a feature which cannot be explained by simple mean-field models [19].

The outline of this paper is as follows: In Sec. [1, we present the effective Lagrangian which contains the relevant baryon-meson vertices. Section $\mathbb{1 1}$ il is devoted to a brief discussion of strangeness-changing response functions. We also discuss the residual particle-hole interaction and the RPA equations. Section $\mathbb{\nabla}$ contains specific results for hypernuclear spectra. Finally, Sec. V contains a short summary. 


\section{THE EFFECTIVE LAGRANGIAN}

To describe the nuclear ground state we use a relativistic mean-filed model based on an effective Lagrangian that realizes chiral symmetry and vector meson dominance. In the nucleon sector this approach has been successful in describing the ground state properties of ordinary nuclei [17]. More recently, these ideas have been generalized to include strangeness 8.

The Lagrangian is formulated in terms of the effective degrees of freedom that are taken to be the baryon octet, the Goldstone boson octet and the vector meson nonet:

$$
\mathcal{L}=\mathcal{L}_{B M}+\mathcal{L}_{M}+\mathcal{L}_{E M}
$$

where the subscript $M(M B)$ denotes the meson (meson-baryon) sector and $E M$ the electromagnetic interaction. The octets are collected in $3 \times 3$ traceless hermitian matrices

$$
\begin{gathered}
B=\left(\begin{array}{ccc}
\frac{1}{\sqrt{6}} \Lambda+\frac{1}{\sqrt{2}} \Sigma^{0} & \Sigma^{+} & p \\
\Sigma^{-} & \frac{1}{\sqrt{6}} \Lambda-\frac{1}{\sqrt{2}} \Sigma^{0} & n \\
\Xi^{-} & \Xi^{0} & -\frac{2}{\sqrt{6}} \Lambda
\end{array}\right), \\
\Pi=\left(\begin{array}{ccc}
\frac{1}{\sqrt{6}} \eta+\frac{1}{\sqrt{2}} \pi^{0} & \pi^{+} & K^{+} \\
\pi^{-} & \frac{1}{\sqrt{6}} \eta-\frac{1}{\sqrt{2}} \pi^{0} & K^{0} \\
K^{-} & \bar{K}^{0} & -\frac{2}{\sqrt{6}} \eta
\end{array}\right), \\
V_{\mu}=\left(\begin{array}{ccc}
\frac{1}{\sqrt{6}} V_{\mu}^{8}+\frac{1}{\sqrt{2}} \rho_{\mu}^{0} & \rho_{\mu}^{+} & K_{\mu}^{*+} \\
\rho_{\mu}^{-} & \frac{1}{\sqrt{6}} V_{\mu}^{8}-\frac{1}{\sqrt{2}} \rho_{\mu}^{0} & K_{\mu}^{* 0} \\
K_{\mu}^{*-} & \bar{K}_{\mu}^{* 0} & -\frac{2}{\sqrt{6}} V_{\mu}^{8}
\end{array}\right) .
\end{gathered}
$$

The physical $\omega$ and $\phi$ mesons arise from the mixing of the $V_{\mu}^{8}$ and the vector meson singlet $S_{\mu}$ via

$$
\begin{aligned}
\omega_{\mu} & =\cos (\theta) S_{\mu}+\sin (\theta) V_{\mu}^{8}, \\
\phi_{\mu} & =\sin (\theta) S_{\mu}-\cos (\theta) V_{\mu}^{8} .
\end{aligned}
$$

We also include a light isoscalar scalar meson $\varphi$ which simulates the exchange of correlated pions and kaons.

The couplings of the mesons to the baryons are contained in

$$
\begin{aligned}
\mathcal{L}_{M B}^{\prime}= & F \operatorname{Tr}\left(\bar{B} i \gamma_{5}[\mathbf{X}, B]\right)+D \operatorname{Tr}\left(\bar{B} i \gamma_{5}\{x, B\}\right) \\
& -g_{F} \operatorname{Tr}(\bar{B}[Y, B])-g_{D} \operatorname{Tr}(\bar{B}\{V, B\})-g_{S} \operatorname{Tr}(\bar{B} \phi B) \\
& -\frac{f_{F}}{4 M} \operatorname{Tr}\left(\bar{B}\left[\sigma_{\mu \nu} V^{\mu \nu}, B\right]\right)-\frac{f_{D}}{4 M} \operatorname{Tr}\left(\bar{B}\left\{\sigma_{\mu \nu} V^{\mu \nu}, B\right\}\right)-\frac{f_{S}}{4 M} \operatorname{Tr}\left(\bar{B} \sigma_{\mu \nu} S^{\mu \nu} B\right),
\end{aligned}
$$

where we disregard terms generated by the covariant derivative of the baryons. The pseudovector couplings of the kaons to the baryons arise from the expansion 


$$
\begin{aligned}
& \Delta_{\mu}=\frac{1}{2}\left(u^{\dagger} \partial_{\mu} u-u \partial_{\mu} u^{\dagger}\right)=\frac{i}{\sqrt{2} f} \partial_{\mu} \Pi+\ldots \\
& \quad \text { for } \quad u=e^{\frac{i}{\sqrt{2} f} \Pi} .
\end{aligned}
$$

For vertices involving kaons $f$ is taken to be the kaon decay constant $f \approx 114.4 \mathrm{MeV}$. The couplings $F$ and $D$ are constrained by $F+D=g_{A}$; for the calculation we use $F=3 / 4$ and $D=1 / 2$.

Couplings to the electromagnetic field are introduced by

$$
\begin{aligned}
\mathcal{L}_{E M}= & -e \operatorname{Tr}(\bar{B}[\mathcal{Q} A, B])-e \frac{\mu_{D}}{4 M} \operatorname{Tr}\left(\bar{B} \sigma_{\mu \nu} F^{\mu \nu}\{\mathcal{Q}, B\}\right)-e \frac{\mu_{F}}{4 M} \operatorname{Tr}\left(\bar{B} \sigma_{\mu \nu} F^{\mu \nu}[\mathcal{Q}, B]\right) \\
& +e \frac{\beta_{D}}{2 M^{2}} \operatorname{Tr}\left(\bar{B} \gamma^{\nu} \partial^{\mu} F_{\mu \nu}\{\mathcal{Q}, B\}\right)+e \frac{\beta_{F}}{2 M^{2}} \operatorname{Tr}\left(\bar{B} \gamma^{\nu} \partial^{\mu} F_{\mu \nu}[\mathcal{Q}, B]\right),
\end{aligned}
$$

where $\mathcal{Q}=\operatorname{diag}\{2 / 3,-1 / 3,-1 / 3\}$ is the quark charge matrix. Combined with vector meson dominance, the Lagrangian Eq. (8) describes the low energy electromagnetic structure of the baryons so that no external form factors are needed.

To constrain the couplings we follow closely Ref. [8]. For the meson-baryon couplings we assume $S U(3)$ symmetry and that the OZI rule holds, i.e. the couplings between nucleons and the $\phi$ meson vanish. Furthermore, relation Eq. (5) is implemented with the ideal mixing angle $\sin (\theta)=1 / \sqrt{3}$. For given values of the corresponding $\omega$ and $\rho$ couplings to the nucleons, the set of parameters $\left(g_{F}, g_{D}, g_{S}, f_{F}, f_{D}, f_{S}\right)$ is then fixed ( $M$ is taken to be the nucleon mass).

The electromagnetic structure generated by Eq. (8) holds only in the strict $S U(3)$ limit. The physical values of the magnetic moments can be generated by adding appropriate symmetry breaking terms. We use the Particle Data Group [20] values

$$
\mu_{\Lambda}=-0.613
$$

For the magnetic moment for the $\Sigma^{0}$, which is experimentally not accessible, we employ the result of the chiral perturbation theory calculation in Ref. 21]

$$
\mu_{\Sigma^{0}}=0.65 .
$$

The parameters $\beta_{F}$ and $\beta_{D}$ contribute to the charge radii of the baryons which are not known in the hyperon sector. For simplicity, we assume $S U(3)$ symmetry which determines the parameters from the corresponding values in the nucleon sector. As discussed later we adjust the coupling of the $\Lambda$ to the scalar field $\varphi$ to obtain a good reproduction of the experimentally known levels in ${ }_{\Lambda}^{16} \mathrm{O}$ [11]. For the $\Sigma^{0}$ we follow the phenomenological approach of Refs. [8,22] and require that the coupling reproduces the hyperon potential in nuclear matter which is taken to be

$$
U^{\Sigma}=g_{\Sigma}^{\varphi} \varphi-g_{\Sigma}^{\omega} \omega^{0} \approx 25 \mathrm{MeV} .
$$

In the nucleon sector we employ the parameter set G1 of Ref. 117. The corresponding hyperon couplings are listed in Table $\mathbb{1}$.

In principle, the Lagrangian Eq. (而) contains vertices that generate a non-diagonal self energy in the $\Lambda-\Sigma^{0}$ sector of flavor space. However, flavor mixing has a very small effect on the hypernuclear energy levels [23] and will be disregarded in the following. 
To generate the ground state of the initial nucleus the meson field operators in the Lagrangian Eq. (11) are replaced by their mean field values. The lowest order response functions are then calculated in a perturbative expansion with respect to this ground state.

\section{STRANGENESS-CHANGING RESPONSE FUNCTIONS}

Among the many elementary processes that may produce a hyperon on a nuclear target our theoretical analysis is guided by the hadronic and photoproduction reactions discussed in Sec. I. Yet rather than focus on the calculation of the cross section, we consider the nuclear response functions themselves, as they contain all essential information on hypernuclear spectra. The linear response of the nuclear ground state to any external probe is related to a suitably defined polarization tensor. The polarization tensor is a fundamental many-body operator that may be computed systematically using well-known many-body techniques, such as Feynman diagrams and Dyson's equation 24].

\section{A. Lowest-order Polarization}

To illustrate the many-body techniques employed here we simplify the model presented in Sect. III and assume that there are no meson-meson self interactions and that there is only one type of hyperons interacting with nucleons and mesons. Without loss of generality we concentrate on the pseudoscalar polarization in the following. This many-body operator is defined as the ground-state expectation value of a time-ordered product of pseudoscalar currents

$$
i \Pi^{5,5}(x, y)=\left\langle\Psi_{0}\left|T\left[J^{5}(x) J^{5}(y)\right]\right| \Psi_{0}\right\rangle
$$

where

$$
J^{5}(x)=\bar{\psi}_{Y}(x) i \gamma^{5} \psi_{N}(x)
$$

In a mean-field approximation to the ground state the polarization insertion can be written exclusively in terms of hyperon and nucleon mean-field propagators

$$
i \Pi^{5,5}(x, y)=\operatorname{Tr}\left[\left(i \gamma^{5}\right) G_{Y}(x, y)\left(i \gamma^{5}\right) G_{N}(y, x)\right] .
$$

These propagators contain information about the interaction of the propagating baryon with the average mean field provided by the nuclear medium. Note that even in a simplified description in which the interactions are ignored, such as in a Fermi-gas treatment, the nucleon - but not the hyperon - propagator would still differ from its free-space value because of the filled Fermi sea. This fact suggests the following decomposition of the nucleon propagator 25]:

$$
\begin{aligned}
& G_{N}(x, y)=\int_{-\infty}^{\infty} \frac{d \omega}{2 \pi} e^{-i \omega\left(x^{0}-y^{0}\right)} G_{N}(\mathbf{x}, \mathbf{y} ; \omega) \\
& G_{N}(\mathbf{x}, \mathbf{y} ; \omega)=G_{F}(\mathbf{x}, \mathbf{y} ; \omega)+G_{D}(\mathbf{x}, \mathbf{y} ; \omega)
\end{aligned}
$$


The Feynman part of the propagator, $G_{F}$, admits a spectral decomposition in terms of the mean-field solutions to the Dirac equation. That is,

$$
G_{F}(\mathbf{x}, \mathbf{y} ; \omega)=\sum_{\alpha}\left[\frac{U_{\alpha}(\mathbf{x}) \bar{U}_{\alpha}(\mathbf{y})}{\omega-E_{\alpha}^{(+)}+i \eta}+\frac{V_{\alpha}(\mathbf{x}) \bar{V}_{\alpha}(\mathbf{y})}{\omega+E_{\alpha}^{(-)}-i \eta}\right],
$$

where $U_{\alpha}$ and $V_{\alpha}$ are the positive- and negative-energy solutions to the Dirac equation, and the sum is over all states in the spectrum. The density-dependent part of the propagator, $G_{D}$, corrects $G_{F}$ for the presence of a filled Fermi surface. Formally, one effects this correction by shifting the position of the pole of every occupied state from below to above the real axis

$$
\begin{aligned}
G_{D}(\mathbf{x}, \mathbf{y} ; \omega) & =\sum_{\alpha<\mathrm{F}} U_{\alpha}(\mathbf{x}) \bar{U}_{\alpha}(\mathbf{y})\left[\frac{1}{\omega-E_{\alpha}^{(+)}-i \eta}-\frac{1}{\omega-E_{\alpha}^{(+)}+i \eta}\right] \\
& =2 \pi i \sum_{\alpha<\mathrm{F}} \delta\left(\omega-E_{\alpha}^{(+)}\right) U_{\alpha}(\mathbf{x}) \bar{U}_{\alpha}(\mathbf{y}) .
\end{aligned}
$$

Note that the sum over $\alpha$ is now restricted to only those positive-energy nucleon states below the Fermi surface. The hyperon propagator, because of the $S=0$ nature of the nuclear ground state, does not suffer from such a correction.

The decomposition of the nucleon propagator into Feynman and density-dependent contributions suggests an equivalent decomposition for the polarization insertion

$$
\begin{aligned}
& \Pi^{5,5}(x, y)=\int_{-\infty}^{\infty} \frac{d \omega}{2 \pi} e^{-i \omega\left(x^{0}-y^{0}\right)} \Pi^{5,5}(\mathbf{x}, \mathbf{y} ; \omega) \\
& \Pi^{5,5}(\mathbf{x}, \mathbf{y} ; \omega)=\Pi_{F}^{5,5}(\mathbf{x}, \mathbf{y} ; \omega)+\Pi_{D}^{5,5}(\mathbf{x}, \mathbf{y} ; \omega)
\end{aligned}
$$

The Feynman part of the polarization, $\Pi_{F}^{5,5}$, describes pair production - or vacuum polarization. In infinite nuclear matter, the threshold for pair production lies well into the timelike region at $q^{2}=\left(M_{Y}^{*}+M_{N}^{*}\right)^{2}\left(M^{*}\right.$ is the effective baryon mass in the nuclear medium). This is far away from the spacelike region accessible in hadronic and photoproduction processes. Thus the lowest-order response - which is proportional to the imaginary part of the polarization insertion - is not sensitive to pair production and will be ignored henceforth. Yet this statement should be taken with caution as we are interested in computing the nuclear response beyond first order. Although the imaginary part of vacuum polarization does indeed vanish for spacelike processes, its dispersive (real) content does not: vacuum excitations can be produced virtually. Thus in more sophisticated treatments of the response than the one presented here, pair production may play a significant role. Still, the RPA response presented here is fully consistent with the "no-vacuum" approximation to the mean-field ground state.

In contrast to the Feynman part of the polarization, which is divergent and must be renormalized, the density-dependent part is finite and given by

$$
\Pi_{D}^{5,5}(\mathbf{x}, \mathbf{y} ; \omega)=\sum_{\alpha<F} \bar{U}_{\alpha}(\mathbf{x})\left(i \gamma^{5}\right) G_{Y}\left(\mathbf{x}, \mathbf{y} ; E_{\alpha}^{(+)}+\omega\right)\left(i \gamma^{5}\right) U_{\alpha}(\mathbf{y})
$$

The density-dependent part of the polarization describes the traditional excitation of particle-hole pairs. This may be most clearly seen by using a spectral decomposition of the hyperon propagator as in Eq. (17) 
$\Pi_{D}^{5,5}(\mathbf{x}, \mathbf{y} ; \omega)=\sum_{\alpha<F, \beta}\left[\frac{\bar{U}_{\alpha}(\mathbf{x})\left(i \gamma^{5}\right) \mathcal{U}_{\beta}(\mathbf{x}) \overline{\mathcal{U}}_{\beta}(\mathbf{y})\left(i \gamma^{5}\right) U_{\alpha}(\mathbf{y})}{\omega-\left(\mathcal{E}_{\beta}^{+}-E_{\alpha}^{+}\right)+i \eta}+\frac{\bar{U}_{\alpha}(\mathbf{x})\left(i \gamma^{5}\right) \mathcal{V}_{\beta}(\mathbf{x}) \overline{\mathcal{V}}_{\beta}(\mathbf{y})\left(i \gamma^{5}\right) U_{\alpha}(\mathbf{y})}{\omega+\left(\mathcal{E}_{\beta}^{-}+E_{\alpha}^{+}\right)-i \eta}\right]$

The first term in the sum represents the formation of a $Y$-particle- $N$-hole pair after the probe has transfered an energy $\omega$ to the nucleus. The excitation of the pair becomes real, namely both particles get on their mass shell, only when the energy transfer is identical to the excitation energy $\omega \equiv \mathcal{E}_{\beta}^{+}-E_{\alpha}^{+}$. That is, the inclusive pseudoscalar response to lowest order becomes

$$
S_{\mathrm{ps}}(\mathbf{q}, \omega)=-\frac{1}{\pi} \operatorname{Im}\left[\Pi_{D}^{5,5}(\mathbf{q}, \mathbf{q} ; \omega)\right]=\sum_{\alpha<F, \beta}\left|\rho_{\beta \alpha}^{(5)}(q)\right|^{2} \delta\left(\omega-\omega_{\beta \alpha}\right) .
$$

Note that we have introduced the transition density $\rho_{\beta \alpha}^{(5)}$ and the excitation energy $\omega_{\beta \alpha}$ by

$$
\rho_{\beta \alpha}^{(5)}(q) \equiv \int d^{3} x e^{i \mathbf{q} \cdot \mathbf{x}} \overline{\mathcal{U}}_{\beta}(\mathbf{x})\left(i \gamma^{5}\right) U_{\alpha}(\mathbf{x}) \quad \text { and } \quad \omega_{\beta \alpha} \equiv \mathcal{E}_{\beta}^{+}-E_{\alpha}^{+}
$$

The second term in the sum is interesting and has no nonrelativistic counterpart; it represents the Pauli blocking of the vacuum excitations discussed earlier. This term mixes positive (nucleon) and negative (hyperon) states and prevents the nucleon — from the $N \bar{Y}$ pair from occupying a state below the Fermi surface. As in the case of vacuum polarization, this term is purely real and makes no contribution to the lowest-order response. Yet its inclusion in the correlated RPA response is essential in order to satisfy fundamental symmetries of nature, such as gauge invariance.

\section{B. The RPA equations}

In our simplified version of the model, the polarization tensor also describes modifications to the propagation of mesons (such as the $K^{-}$meson), in addition to containing all information about the excitation spectra of hypernuclei. In the lowest-order approximation, the residual interaction between the particle and the hole is neglected. A consistent approximation scheme that goes beyond lowest order — by including the residual interaction between the nucleon-hole and the hyperon-particle is the random-phase approximation. In the RPA one includes many-body correlations by iterating to infinite order the lowest-order polarization insertion. For example, assuming a pseudo-scalar coupling, this means

$$
\Pi_{\mathrm{RPA}}^{5,5}\left(\mathbf{q}, \mathbf{q}^{\prime} ; \omega\right)=\Pi^{5,5}\left(\mathbf{q}, \mathbf{q}^{\prime} ; \omega\right)+\int \frac{d^{3} k}{(2 \pi)^{3}} \Pi^{5,5}(\mathbf{q}, \mathbf{k} ; \omega) V_{K}(\mathbf{k} ; \omega) \Pi_{\mathrm{RPA}}^{5,5}\left(\mathbf{k}, \mathbf{q}^{\prime} ; \omega\right)
$$

where we have introduced the free kaon propagator through

$$
V_{K}(\mathbf{k} ; \omega)=-g_{Y N K}^{2} \Delta_{K}(\mathbf{k}, \omega)=\frac{-g_{Y N K}^{2}}{\omega^{2}-\mathbf{k}^{2}-m_{K}^{2}} .
$$

Note that $g_{Y N K}$ represents the hyperon-nucleon-kaon coupling constant. That the RPA is the consistent linear response of the mean-field ground state has been amply demonstrated 
through the excitation of the isoscalar-dipole $\left(J^{\pi}=1^{-} ; T=0\right)$ mode. The isoscalar-dipole response provides a delicate test of the self-consistency of the calculation. Indeed, a calculation of the isoscalar-dipole response to lowest-order is flawed by the presence of spurious strength associated with the uniform translation of the center of mass. In contrast, a consistent RPA calculation - one that uses the same interaction in the calculation of the mean-field ground-state than in the calculation of the response - eliminates this anomalous behavior by shifting all spurious strength to zero excitation energy.

In the previous section a spectral decomposition of the Feynman propagator was presented. Although such a decomposition is useful for understanding the spectral content of the nuclear response, in practice it suffers from too much reliance on artificial cutoffs. An efficient scheme that avoids the introduction of artificial cutoffs is the non-spectral approach. Moreover, a non-spectral formalism has the added advantage that, at least in principle, the positive-energy continuum may be treated exactly. The non-spectral generation of the Feynman propagator amounts to solving the following inhomogeneous Dirac equation

$$
\left[\omega \gamma^{0}+i \gamma \cdot \nabla-M_{B}-\Sigma(\mathbf{x})\right] G_{F}(\mathbf{x}, \mathbf{y} ; \omega)=\delta(\mathbf{x}-\mathbf{y})
$$

where $\omega$ is in general a complex variable and the mean-field potential, or baryon self-energy, is given by

$$
\Sigma(\mathbf{x})=\Sigma_{s}(\mathbf{x})+\gamma^{0} \Sigma_{0}(\mathbf{x})-\frac{i}{2 M} \gamma^{0} \frac{\gamma \cdot \mathbf{x}}{|\mathbf{x}|} \Sigma_{T}(\mathbf{x})
$$

The quantities $\Sigma_{s}, \Sigma_{0}$ and $\Sigma_{T}$ contain the nuclear scalar, vector and tensor potentials generated at the mean-field level. These mean-field self-energies have been collected in Table II. Note that we have assumed that the mean-field potential is generated by a sphericallysymmetric, spin-saturated ground state. Taking advantage of this spherical symmetry one may decompose the Feynman propagator in terms of spin-spherical harmonics

$$
G_{F}(\mathbf{x}, \mathbf{y} ; \omega)=\frac{1}{x y} \sum_{\kappa m}\left(\begin{array}{rr}
g_{11}^{\kappa}(x, y ; \omega)\langle\hat{\mathbf{x}}++\kappa m\rangle\langle+\kappa m \mid \hat{\mathbf{y}}\rangle & -i g_{12}^{\kappa}(x, y ; \omega)\langle\hat{\mathbf{x}} \mid+\kappa m\rangle\langle-\kappa m \mid \hat{\mathbf{y}}\rangle \\
i g_{21}^{\kappa}(x, y ; \omega)\langle\hat{\mathbf{x}} \mid-\kappa m\rangle\langle+\kappa m \mid \hat{\mathbf{y}}\rangle & g_{22}^{\kappa}(x, y ; \omega)\langle\hat{\mathbf{x}} \mid-\kappa m\rangle\langle-\kappa m \mid \hat{\mathbf{y}}\rangle
\end{array}\right)
$$

which are defined as

$$
\begin{gathered}
\langle\hat{\mathbf{x}} \mid \kappa m\rangle=\sum_{m_{l} m_{s}}\left\langle l m_{l}, \frac{1}{2} m_{s} \mid l \frac{1}{2} j m\right\rangle Y_{l m_{l}}(\hat{\mathbf{x}}) \chi_{\frac{1}{2} m_{s}}, \\
j=|\kappa|-\frac{1}{2} \quad \text { and } \quad l= \begin{cases}+\kappa & \text { if } \kappa>0, \\
-\kappa-1 & \text { if } \kappa<0 .\end{cases}
\end{gathered}
$$

The above decomposition enables one to rewrite the Dirac equation as a set of first-order, coupled, ordinary differential equations of the form

$$
\left(\begin{array}{cc}
\omega^{*}-M^{*} & \frac{d}{d x}-\frac{\kappa^{*}}{x} \\
\frac{d}{d x}+\frac{\kappa^{*}}{x} & -\omega^{*}-M^{*}
\end{array}\right)\left(\begin{array}{cc}
g_{11}^{\kappa} & g_{12}^{\kappa} \\
g_{21}^{\kappa} & g_{22}^{\kappa}
\end{array}\right)=\delta(x-y),
$$

where we have defined 


$$
\omega^{*} \equiv \omega-\Sigma_{v}(x), M^{*} \equiv M_{B}+\Sigma_{s}(x), \text { and } \quad \kappa^{*} \equiv \kappa-\frac{x}{2 M} \Sigma_{T}(x) .
$$

Similarly, a positive-energy Dirac spinor

$$
U_{\alpha}(\mathbf{x})=\frac{1}{x}\left(\begin{array}{c}
g_{n \kappa}(x)\langle\hat{\mathbf{x}} \mid+\kappa m\rangle \\
i f_{n \kappa}(x)\langle\hat{\mathbf{x}} \mid-\kappa m\rangle
\end{array}\right) \quad(\alpha \equiv n \kappa m)
$$

satisfies the homogeneous Dirac equation

$$
\begin{aligned}
& \left(\frac{d}{d x}+\frac{\kappa^{*}}{x}\right) g_{n \kappa}(x)-\left(E^{*}+M^{*}\right) f_{n \kappa}(x)=0, \\
& \left(\frac{d}{d x}-\frac{\kappa^{*}}{x}\right) f_{n \kappa}(x)+\left(E^{*}-M^{*}\right) g_{n \kappa}(x)=0 .
\end{aligned}
$$

At the beginning of this section we expressed Dyson's equation for the pseudoscalar polarization as an integral equation in three dimensions [see Eq. (26)]. Yet the spherical symmetry of the problem simplifies this numerical task considerably. To do so we used the results derived earlier for the Feynman propagator and for the positive-energy Dirac spinors to perform a multipole decomposition of the polarization insertion as described in Ref. [26]:

$$
\Pi^{5,5}\left(\mathbf{q}, \mathbf{q}^{\prime} ; \omega\right)=\sum_{J=0}^{\infty} \Pi_{J}^{5,5}\left(q, q^{\prime} ; \omega\right) P_{00}^{J}\left(\hat{\mathbf{q}}, \hat{\mathbf{q}}^{\prime}\right)
$$

Here the angular dependence is fully contained in the function,

$$
P_{\lambda \lambda^{\prime}}^{J}\left(\hat{\mathbf{q}}, \hat{\mathbf{q}}^{\prime}\right)=\sum_{M} D_{M \lambda}^{J}(\hat{\mathbf{q}}) D_{M \lambda^{\prime}}^{J *}\left(\hat{\mathbf{q}}^{\prime}\right)
$$

which is expressed in terms of Wigner D-functions [27]. The multipoles of the polarization insertion, $\Pi_{J}^{5,5}$, involve various reduced matrix elements enforcing selection rules for the intermediate hyperon-particle and nucleon-hole states that couple to a total angular momentum $J$. Note that because of the pseudoscalar nature $\left(\gamma_{5}\right)$ of the vertex, unnatural parity states $\left(J^{\pi}=0^{-}, 1^{+}, \ldots\right)$ are created exclusively. One can easily show, as it has been done Ref. [26], that by using the following identity

$$
\int d \hat{\mathbf{k}} P_{\lambda \sigma}^{J}(\hat{\mathbf{q}}, \hat{\mathbf{k}}) P_{\sigma \lambda^{\prime}}^{J^{\prime}}\left(\hat{\mathbf{k}}, \hat{\mathbf{q}}^{\prime}\right)=\frac{4 \pi}{2 J+1} \delta_{J J^{\prime}} P_{\lambda \lambda^{\prime}}^{J}\left(\hat{\mathbf{q}}, \hat{\mathbf{q}}^{\prime}\right)
$$

the three-dimensional integral equation for the RPA polarization [Eq. (26)] can now be reduced to a one-dimensional one, albeit one for each value of $J$ :

$$
\Pi_{\mathrm{RPA}, J}^{5,5}\left(q, q^{\prime} ; \omega\right)=\Pi_{J}^{5,5}\left(q, q^{\prime} ; \omega\right)+\frac{1}{2 J+1} \int \frac{d k}{2 \pi^{2}} \Pi_{J}^{5,5}(q, k ; \omega) V_{K}(k ; \omega) \Pi_{\mathrm{RPA}, J}^{5,5}\left(k, q^{\prime} ; \omega\right) .
$$




\section{Residual particle-hole interaction}

The RPA equation (26) is only applicable in the simplified version of the hadronic model where the full response function Eq.(12) is equivalent to the full kaon propagator. In general, the connection between response functions and full meson propagators is more involved because of kaon and pion self-interactions and because of the fact that different hyperon flavors can couple to the same external source. Taking into account the possibility of creating a particle-hole state with a $\Lambda$ or a $\Sigma^{0}$ hyperon we consider response functions of the type

$$
\Pi^{\alpha, \beta}(x, y)=\sum_{Y=\Lambda, \Sigma^{0}}\left\langle\Psi_{0}\left|T\left[\bar{\Psi}_{N}(x) \Gamma_{Y}^{\alpha} \Psi_{Y}(x) \bar{\Psi}_{Y}(y) \Gamma_{Y}^{\beta} \Psi_{N}(y)\right]\right| \Psi_{0}\right\rangle
$$

where $\Gamma_{Y}^{\alpha}$ characterizes specific interaction vertices. Although these response functions can be analyzed for arbitrary vertices in terms of Feynman diagrams it is more intuitive to cast the problem into a form similar to Eq. (26). In analogy to Eq. (21) we keep only the density-dependent part for the lowest order contribution to the response function (40). As a further approximation we neglect internal vertices that give rise to meson-meson self interactions and loops involving $\Sigma^{ \pm}$and $\Xi^{\overline{0}}$ baryons. These contributions constitute pure "vacuum" contributions as discussed in Sec. IIIA. The remaining piece to be specified is then the residual hyperon-nucleon interaction. Although considerable progress has been made towards elucidating the precise form of the interaction, much work remains to be done. Thus in this, our first contribution to the subject, we make a very simple assumption as to the nature of the hyperon-nucleon residual interaction. We assume, in analogy with the " $\pi+\rho "$ isovector interaction, that the residual interaction in the hyperon-nucleon channel is mediated by the two lightest $S=-1$ mesons: the pseudoscalar kaon and its vector partner the $K^{*}(892)$. In this case the range of the interaction is determined from the free propagators. These are given by

$$
\begin{aligned}
\Delta_{K}(q) & =\frac{1}{q^{2}-m_{K}^{2}}, \\
D_{K^{*}}^{\mu \nu}(q) & =\frac{-g^{\mu \nu}+q^{\mu} q^{\nu} / m_{K^{*}}^{2}}{q^{2}-m_{K^{*}}^{2}} .
\end{aligned}
$$

The strength and the spin structure of the residual interaction is, on the other hand, determined by the vertices. For the $Y N K$ vertex we assume a pseudovector - as opposed to a pseudoscalar - representation, as detailed in Eq. (6). Although both representations are equivalent on-shell, it is convenient to adopt the pseudovector representation as it accounts for the correct low-energy theorems - especially in the case of the pion, the lightest member of the pseudoscalar octet — without the need for sensitive cancellations. The $K^{*}$-meson contains a vector as well as a tensor coupling to the baryons. These choices specify completely the nature of the elementary $Y N K$ and $Y N K^{*}$ vertices:

$$
\Gamma_{Y}^{\alpha}= \begin{cases}\frac{g_{Y N}^{K}}{f_{K}} \phi \gamma^{5} & \text { if } \alpha=K \\ g_{Y N}^{K^{*}} \gamma^{\mu}+i f_{Y N}^{K^{*}} \sigma_{\mu \nu} \frac{q_{\nu}}{2 M} & \text { if } \alpha=K^{*} .\end{cases}
$$

Choosing $\alpha=\beta=K$ and $\alpha=\beta=K^{*}$ in Eq. (40) defines a $K$ and a $K^{*}$ response function, respectively. With the approximation outlined above, each response functions can then be obtained from a RPA equations similar to Eq. (26). 
The spin structure of the vertices is well known, at least in the nonrelativistic limit. Indeed, as in the case of the pion, the kaon generates a spin-longitudinal coupling of the form

$$
\frac{g_{Y N}^{K}}{f_{K}} \not \gamma^{5} \rightarrow \frac{g_{Y N}^{K}}{f_{K}}(\sigma \cdot \mathbf{q}) .
$$

Thus, the kaon excites exclusively hypernuclear states of unnatural parity. In contrast, the vector $K^{*}$-meson induces, in addition to the simple spin-independent coupling stemming from the timelike part of Dirac vertex, a spin-transverse coupling of the form

$$
\begin{aligned}
& g_{Y N}^{K^{*}} \gamma \rightarrow \frac{g_{Y N}^{K^{*}}}{2 M}(\sigma \times \mathbf{q}), \\
& f_{Y N}^{K^{*}} \sigma_{\mu \nu} \frac{q_{\nu}}{2 M} \rightarrow \frac{f_{Y N}^{K^{*}}}{2 M}(\sigma \times \mathbf{q}) .
\end{aligned}
$$

Hence, with the exception of $0^{-}$states, a $K^{*}$-meson can excite hypernuclear states of all spins and parities. Note that because of the present lack of theoretical guidance, no repulsive shortrange Landau-Migdal parameter has been introduced. Thus, in this simplified description there is no source of mixing between the spin-longitudinal and the spin-transverse modes.

\section{RESULTS AND DISCUSSION}

Let us start the discussion with the $K^{*}$ response and assume that the hypernuclear states are created by replacing a neutron in the initial nucleus with a hyperon. Although the calculated response function contains information on both $\Lambda$ and $\Sigma^{0}$ states, we will restrict the discussion to energies well below the threshold for creating $\Sigma^{0}$ hypernuclei.

In analogy to the electromagnetic case, the $K^{*}$ response can be analyzed in terms of structure functions. Unlike the electromagnetic response, however, there are generally four structure functions because the $K^{*}$ does not couple to a conserved current. Here we focus on the longitudinal and transverse response which are defined by

$$
\begin{aligned}
& \Pi_{L}\left(q_{0}, \mathbf{q}, \mathbf{q}\right)=\Pi^{00}\left(q_{0}, \mathbf{q}, \mathbf{q}\right) \\
& \Pi_{T}\left(q_{0}, \mathbf{q}, \mathbf{q}\right)=\Pi_{i}^{i}\left(q_{0}, \mathbf{q}, \mathbf{q}\right)-\frac{q_{i} q_{j}}{|\mathbf{q}|} \Pi^{i j}\left(q_{0}, \mathbf{q}, \mathbf{q}\right)
\end{aligned}
$$

and that contain all the relevant information on the spectra.

In hadronic reactions of the type $\left(K^{-}, \pi^{-}\right)$hypernuclear states are effectively populated if the outgoing meson is emitted in the forward direction. Consequently, these reactions are characterized by small momentum transfer $\left(|\mathbf{q}|<q_{0}\right)$. The RPA results for the longitudinal $K^{*}$ response in oxygen are indicated in Fig.1. At low momentum transfer $(|\mathbf{q}|=50 \mathrm{MeV})$ the multipole expansion is rapidly converging and only the lowest order terms are indicated. The coupling of the scalar $\varphi$-meson was adjusted to reproduce the two $0^{+}$and $1^{-}$states that are experimentally identified in the ${ }^{16} \mathrm{O}\left(K^{-}, \pi^{-}\right){ }_{\Lambda}^{16} \mathrm{O}$ reaction [11. According to Eq. (23), we added a small imaginary part $(i \eta=i .2 \mathrm{MeV})$ to the energy variable to keep the magnitude of the resonances finite. In the notation of Eq. (24), the binding energy $B_{\Lambda}$ of a $\Lambda$ at the location of a resonance may be obtained from 


$$
\omega_{\Lambda 0}=\mathcal{E}_{\Lambda}^{(+)}-E_{0}^{(+)} \equiv B_{n}-B_{\Lambda}+M_{\Lambda}-M_{N}
$$

where $B_{n}$ denotes the separation energy of the weakest bound neutron.

The differences between the RPA and the uncorrelated mean field predictions can be studied in Fig.2 for the $J=0$ multipole. The uncorrelated states are indicated by their particle-hole contents. The resulting RPA states are an admixture of all states with a given total angular momentum $J$. The increase of the hypernuclear binding energies indicates the repulsive character of the residual interaction. Most remarkably, there is no clear remnant of the $\left(1 s_{1 / 2}, 1 s_{1 / 2}^{-1}\right)$ state which has moved into the continuum. This is an important feature because this state is predicted by the simple particle-hole model but is not recognized in the experimental data.

The longitudinal response leads exclusively to states with natural parity. Excitations with unnatural parity arise in the transverse response which is indicated in Fig. 3. In analogy to the electromagnetic case, transverse modes with unnatural parity can be interpreted as magnetic excitations, whereas the modes in Fig. 3 with opposite parity are of transverseelectric character. Compared to the longitudinal response, the strength of the transverse excitations is much smaller. Furthermore, correlation effects are modest as a consequence of the spin-transverse coupling indicated in Eq. (44b).

The RPA predictions for calcium are shown in Fig. 4. The experimental spectrum [28] observed in the $\left(K^{-}, \pi^{-}\right)$reaction on ${ }^{40} \mathrm{Ca}$ exhibits three peaks. Two at $\mathcal{E}_{\Lambda}^{(+)}-E_{0}^{(+)}=194$ and $189 \mathrm{MeV}$ which in the simple particle-hole picture are identified as $0^{+}$states with the particlehole assignments $\left(1 d_{5 / 2}, 1 d_{5 / 2}^{-1}\right)_{\Lambda n}$ and $\left(1 d_{3 / 2}, 1 d_{3 / 2}^{-1}\right)_{\Lambda n}$, respectively. Furthermore, a peak at $180 \mathrm{MeV}$ which is identified as a $1^{-}$state with the assignment $\left(1 p_{1 / 2}, 1 d_{3 / 2}^{-1}\right)_{\Lambda n}$. However, because of the strong mixing it is not possible to attribute the peaks to specific states if correlations are taken into account. To make contact with the experimental observations Fig. 5 shows the difference between the mean field result and the RPA prediction. We remind the reader that the very small width of the states is an idealization and that in a more realistic scenario the peaks are considerably broader. It can be expected that the two group of states around $191 \mathrm{MeV}$ and $197 \mathrm{MeV}$ in the RPA coalesce to form the resonance structure found in the experimental data. Similar as in the case of oxygen, the remnant of the particle-hole state with the neutron in the deepest bound $s$ shell moves into the continuum.

Based on hadronic reactions, transverse modes, including states of unnatural parity, are of minor importance in hypernuclear spectroscopy. Because of their small strength, these states are usually not observed in the kinematic range covered by the experiments [29]. As an attractive alternative, states of unnatural parity may be studied in electromagnetic production of hypernuclei. In our approach unnatural parity states can exclusively be studied in the kaon response function. Under the assumption that the hypernuclei states are created in hyperon photoproduction off a proton, a different range for the momentum transfer has to be considered. With the kinematics forced on the problem the momenta are generally much higher than in the hadronic reactions. However, for the outgoing kaon in forward direction the momentum transfer decreases with increasing photon energy [12 and $|\mathbf{q}|<300 \mathrm{MeV}$ is sufficient. The RPA results for initial oxygen and calcium nuclei are indicated in Fig.6 and Fig.7 at $|\mathbf{q}|=200 \mathrm{MeV}$. Consistent with the observation for the unnatural parity states in the transverse $K^{*}$ response, the residual interaction is rather weak. This can be studied in 
Fig. 8 which compares the mean field and RPA predictions for $J=1$. The labels indicate the particle-hole states on the mean field level. Because of the very weak spin-orbit force felt by the hyperons, the states with the $\Lambda$ in the $1 p_{1 / 2}$ or $1 p_{3 / 2}$ are almost degenerate and coalesce in the figure.

Although the results for the kaon response are consistent with the observations for the transverse $K^{*}$ response, a more unified treatment is desirable. Going beyond our first contribution to the subject, one must allow for mixing between spin-longitudinal and spintransverse modes. Either on a phenomenological level by introducing a Landau-Migdal parameter, or by incorporating higher-order Feynman diagrams, this will be an important topic for future investigations.

\section{CONCLUSIONS}

In this paper we studied strangeness-changing response functions as an alternative approach to hypernuclear structure. In contrast to the traditional mean-field description, where hypernuclear states are uncorrelated hyperon-particle - nucleon-hole excitations, they are treated as any other nuclear excitation that emerges from the response of normal nuclei to external probes. From this point of view we studied hypernuclear spectra using a relativistic random-phase approximation based on a chiral Lagrangian which successfully reproduces properties of normal nuclei and where all relevant meson-baryon vertices are constrained by $\mathrm{SU}(3)$-flavor symmetry.

The response of the nuclear ground state involves several polarization insertions describing the propagation of a nucleon-hole and a $\Lambda\left(\Sigma^{0}\right)$-particle through the nuclear medium. To specify the residual particle-hole interaction we assumed that it is mediated by the two lightest $S=-1$ mesons, namely the kaon and its vector partner the $K^{*}(892)$. This lead us to introduce two strangeness-changing response functions. A vector $K^{*}$ response and a pseudo-vector kaon response. Many-body correlations are included by iterating the lowestorder response to infinite order. The primary difference of the two response functions lies in the structure of their excitation spectra. Due to pseudo-vector character of the $Y N K$ vertex, the kaon exclusively excites hypernuclear states of unnatural parity. In contrast, the $K^{*}$ response contains - with the exception of the $J^{\pi}=0^{-}$state - hypernuclear states of all spin and parities.

We analyzed hypernuclear spectra for ${ }^{16} \mathrm{O}$ and ${ }^{40} \mathrm{Ca}$ nuclei under the assumption that a nucleon in the ground state is replaced by a $\Lambda$ particle. For the longitudinal $K^{*}$ response, which is primarily determined in the hadronic strangeness-exchange reactions such as $\left(K^{-}, \pi^{-}\right)$and $\left(\pi^{+}, K^{+}\right)$, the RPA leads to important corrections over the simple particlehole predictions. First, the repulsive character of the residual interaction decreases the uncorrelated hypernuclear binding energies considerably. This generates the characteristic "quenching and hardening" of the response. Second, we observed important qualitative corrections in the spectra, as the strong residual interaction mixes single-particles states of different particle-hole content but of the same spin and parity. Consequently, in most cases it is no longer possible to attribute the excitations to a specific particle-hole transition. Furthermore, states with nucleons holes in the deepest bound nucleon shell move into the continuum consistent with the experimental observation. In contrast to the longitudinal response, correlation effects are modest in the transverse part of the $K^{*}$ response. 
We also examined the pseudo-vector kaon response responsible for generating hypernuclear states of unnatural parity. In this first study of the spin-longitudinal modes we observed small corrections over the simple particle-hole picture, in analogy to our observations for the spin-transverse $K^{*}$ response. In the future we plan to study this kaonic response in a much more comprehensive way. Our goal will be to establish a dynamical range for which the kaonic enhancement - a precursor to the kaon-condensed state - may be observed. To this end the upcoming nuclear $K^{+}$-photoproduction experiments at TJANF may be of direct relevance to our quest [30]. Indeed, by measuring an enhancement of the photoproduction background, relative to that from a single-nucleon, these experiments may shed light on the exotic and novel states of matter speculated to exist at the core of neutron stars.

\section{ACKNOWLEDGMENTS}

We are pleased to thank J. R. Shepard for useful discussions and comments. This work was supported in part by U.S.DOE under Grant Nos. DE-FG03-93ER-40774, DE-FC0585ER250000 and DE-FG05-92ER40750. 


\section{REFERENCES}

[1] J. Ashman et. al., Phys. Lett. 206B (1988) 364; Nucl. Phys. B328 (1989) 1.

[2] Two excellent recent reviews on strange-quark matter are: Carsten Greiner and Jürgen Schaffner-Bielich, "Physics of Strange Matter", nucl-th/9801062; Jes Madsen, "Physics and Astrophysics of Strange Quark Matter", astro-ph/9809032.

[3] H. Bandō, T. Motoba and J. Žofka, Int. J. of Mod. Phys. A 5 (1990) 4021.

[4] M. M. Nagels, T. A. Rijken and J. J. de Swart, Phys. Rev. D 17 (1978) 768.

P. M. M. Maessen, T. A. Rijken and J. J. de Swart, Phys. Rev. C 40 (1989) 2226.

[5] B. Holzenkamp, K. Holinde and J. Speth, Nucl. Phys. A 500 (1989) 485.

A. Reuber, K. Holinde, H.-C. Kim and J. Speth, Nucl. Phys. A 608 (1996) 243.

[6] J. Schaffner, C. B. Dover, A. Gal, C. Greiner, and H. Stöcker, Phys. Rev. Lett. 71 (1993) 1328.

[7] P. Papazoglou, S. Schramm, J. Schaffner-Bielich, H.Stöcker and W. Greiner, Phys. Rev. C 57 (1998) 2576.

[8] H. Müller, Phys. Rev. C 59 (1999) 1405.

[9] M. Rayet, Nucl. Phys. A 367 (1981) 381.

[10] M. Rufa, H. Stöcker, J. Maruhn, W. Greiner, and P. -G. Reinhard, J. Phys. G 13 (1987) L143.

J. Mareš and J. Žofka, Z. Phys. A 333 (1989) 209.

[11] W. Brückner et al., Phys. Lett. 62B (1976) 481.

[12] A. M. Bernstein, T. W. Donnelly and G. N. Epstein, Nucl. Phys. A 358 (1981) 195c.

[13] J. Cohen, Int. J. of Mod. Phys. A 4 (1989) 1.

[14] C. Bennhold and L. E. Wright, Phys. Rev. C 39 (1989) 927.

[15] J. Hüfner, S. Y. Lee and H. A. Weidenmüller, Nucl. Phys. A 234 (1974) 429.

[16] C. B. Dover, L. Ludeking and G. E. Walker, Phys. Rev. C 22 (1980) 2073.

[17] R. J. Furnstahl, B. D. Serot, and H.-B. Tang, Nucl. Phys. A 615 (1997) 441.

[18] H. C. Chiang and J. Hüfner, Phys. Lett. 84B (1979) 393.

[19] J. Hüfner, S. Y. Lee and H. A. Weidenmüller, Phys. Lett. 49B (1974) 409.

[20] C. Caso et al., The European Physical Journal C 31 (1998).

[21] U.-G. Meißner and S. Steininger, Nucl. Phys. B 499 (1997) 349.

[22] J. Schaffner, C. B. Dover, A. Gal, C. Greiner, D. J. Millener and H. Stöcker, Ann. Phys. (N.Y.) 235 (1994) 35.

[23] H. Müller and J. R. Shepard, nucl-th/9907079 (1999).

[24] A. L. Fetter and J. Di Walecka, "Quantum Theory of Many-Particle Systems", (McGraw-Hill, New York, 1971).

[25] B.D. Serot and J.D. Walecka, Adv. in Nucl. Phys. 16, J.W. Negele and E. Vogt, eds. (Plenum, N.Y. 1986); Int. Jour. Mod. Phys. E 6 (1997) 515.

[26] C. J. Horowitz and J. Piekarewicz, Nucl. Phys. A 511 (1990) 461.

[27] A. R. Edmonds, Angular momentum in quantum mechanics, (Princeton University Press, Princeton, 1957).

[28] B. Povh, Nucl. Phys. A 335 (1990) 233.

[29] R. E. Chrien, et al., Phys. Lett. 89B (1979) 31.

[30] C. Hyde-Wright, Quasifree Strangeness Production in Nuclei, Hall B, Experiment Number E-91-014. 


\section{TABLES}

TABLE I. Hyperon coupling constants.

\begin{tabular}{|c|c|c|c|}
\hline$g_{\Lambda}^{\omega}$ & 7.9125 & $g_{\Sigma}^{\omega}$ & 8.2582 \\
\hline$g_{\Lambda}^{\varphi}$ & 6.0233 & $g_{\Sigma}^{\varphi}$ & 6.0753 \\
\hline$f_{\Lambda}^{\omega}$ & -4.7597 & $f_{\Sigma}^{\omega}$ & 11.739 \\
\hline$g_{\Lambda p}^{K}$ & 0.7939 & $g_{\Sigma p}^{K}$ & 0.1250 \\
\hline$g_{\Lambda n}^{K^{*}}$ & -7.3016 & $g_{\Sigma n}^{K^{*}}$ & 3.8698 \\
\hline$f_{\Lambda n}^{K^{*}}$ & -17.311 & $f_{\Sigma n}^{\bar{K}^{*}}$ & -6.5049 \\
\hline
\end{tabular}

TABLE II. Vector and tensor potentials. $V^{0}$ and $b^{0}$ are the time like component of the $\omega$ and the $\rho^{0}$ mean field respectively, and $A^{0}$ is the Coulomb potential. The couplings of the nucleons to the electromagnetic field are incorporated via the anomalous magnetic moments $\lambda_{p}=1.7928$ and $\lambda_{n}=-1.9131$ and via $\beta_{F}=-0.08230$ and $\beta_{D}=-0.41754$.

\begin{tabular}{|c|c|c|c|c|c|}
\hline \hline$\Sigma_{s}^{p}$ & $-g_{N}^{\varphi} \varphi$ & $\Sigma_{0}^{p}$ & $g_{N}^{\omega} V^{0}+e A^{0}+\frac{1}{2} g_{N}^{\rho} b^{0}+\frac{e}{2 M}\left(\beta_{F}+\frac{1}{3} \beta_{D}\right) \Delta A^{0}$ & $\Sigma_{T}^{p}$ & $\frac{d}{d r}\left(f_{N}^{\omega} V^{0}+\frac{1}{2} f_{N}^{\rho} b^{0}+e \lambda_{p} A^{0}\right)$ \\
$\Sigma_{s}^{n}$ & $-g_{N}^{\varphi} \varphi$ & $\Sigma_{0}^{n}$ & $g_{N}^{\omega} V^{0}-\frac{1}{2} g_{N}^{\rho} b^{0}-\frac{e}{3 M} \beta_{D} \Delta A^{0}$ & $\Sigma_{T}^{n}$ & $\frac{d}{d r}\left(f_{N}^{\omega} V^{0}-\frac{1}{2} f_{N}^{\rho} b^{0}+e \lambda_{n} A^{0}\right)$ \\
$\Sigma_{s}^{\Lambda}$ & $-g_{\Lambda}^{\varphi} \varphi$ & $\Sigma_{0}^{\Lambda}$ & $g_{\Lambda}^{\omega} V^{0}-\frac{e}{6 M} \beta_{D} \Delta A^{0}$ & $\Sigma_{T}^{\Lambda}$ & $\frac{d}{d r}\left(f_{\Lambda}^{\omega} V^{0}+e \mu_{\Lambda} A^{0}\right)$ \\
$\Sigma_{s}^{\Sigma}$ & $-g_{\Sigma^{\varphi}}^{\varphi}$ & $\Sigma_{0}^{\Sigma^{0}}$ & $g_{\Sigma^{0}}^{\omega} V^{0}+\frac{e}{6 M} \beta_{D} \Delta A^{0}$ & $\Sigma_{T}^{\Sigma^{0}}$ & $\frac{d}{d r}\left(f_{\Sigma^{0}}^{\omega} V^{0}+e \mu_{\Sigma^{0}} A^{0}\right)$ \\
\hline \hline
\end{tabular}




\section{FIGURES}

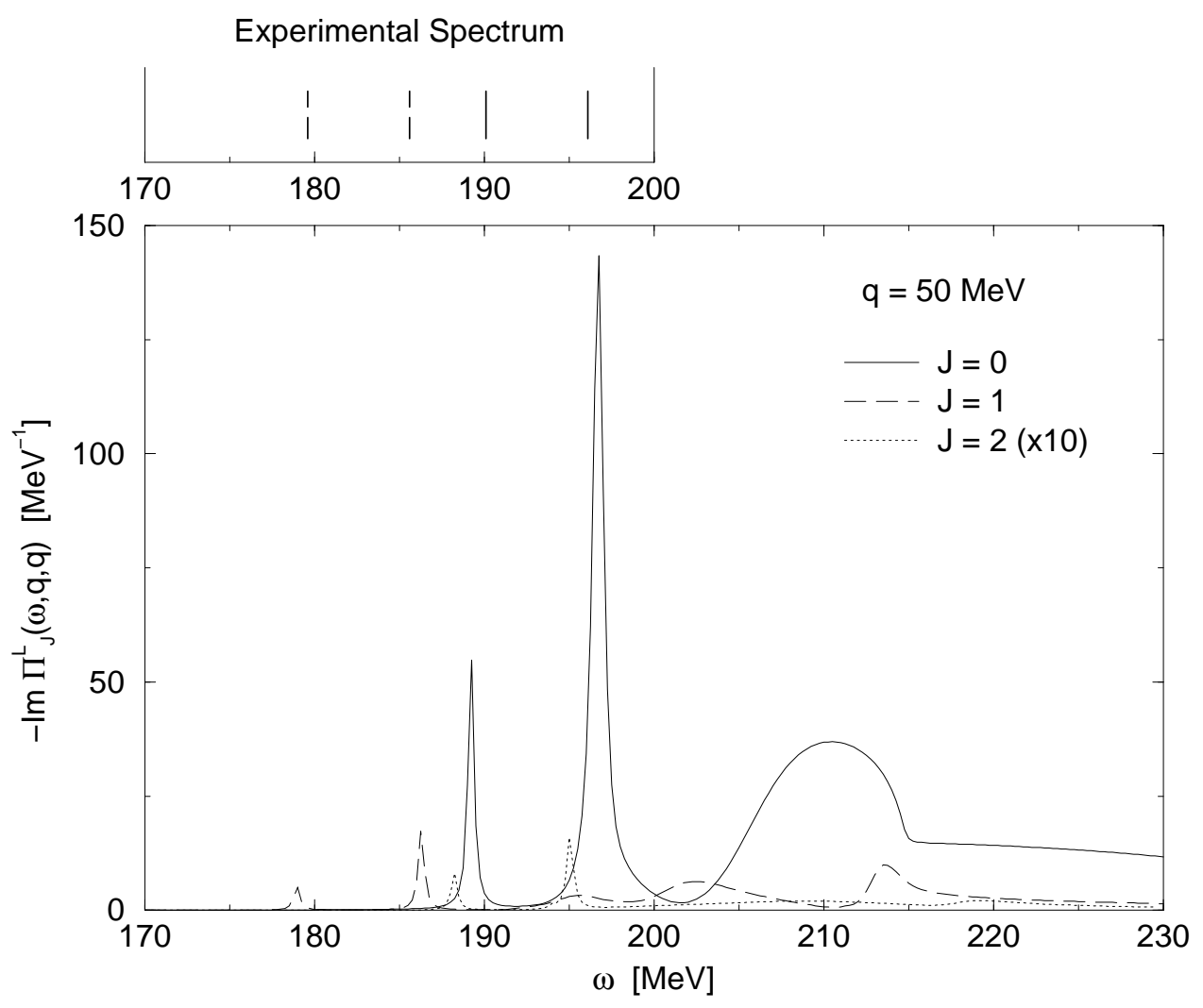

FIG. 1. Lowest order multipoles of the longitudinal $K^{*}$ response in oxygen. 


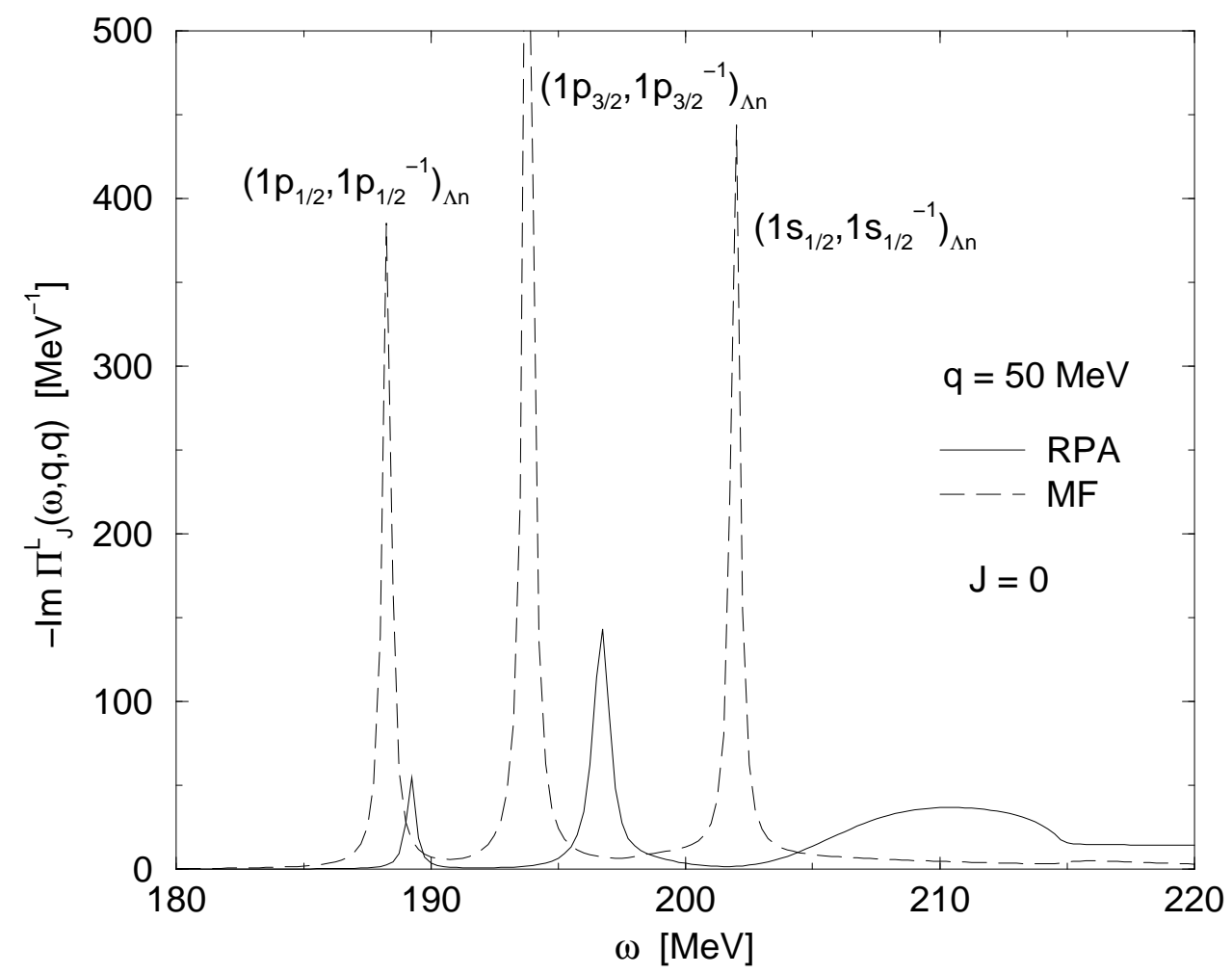

FIG. 2. Mean field and RPA result for the $J=0$ multipole of the longitudinal $K^{*}$ response in oxygen. 


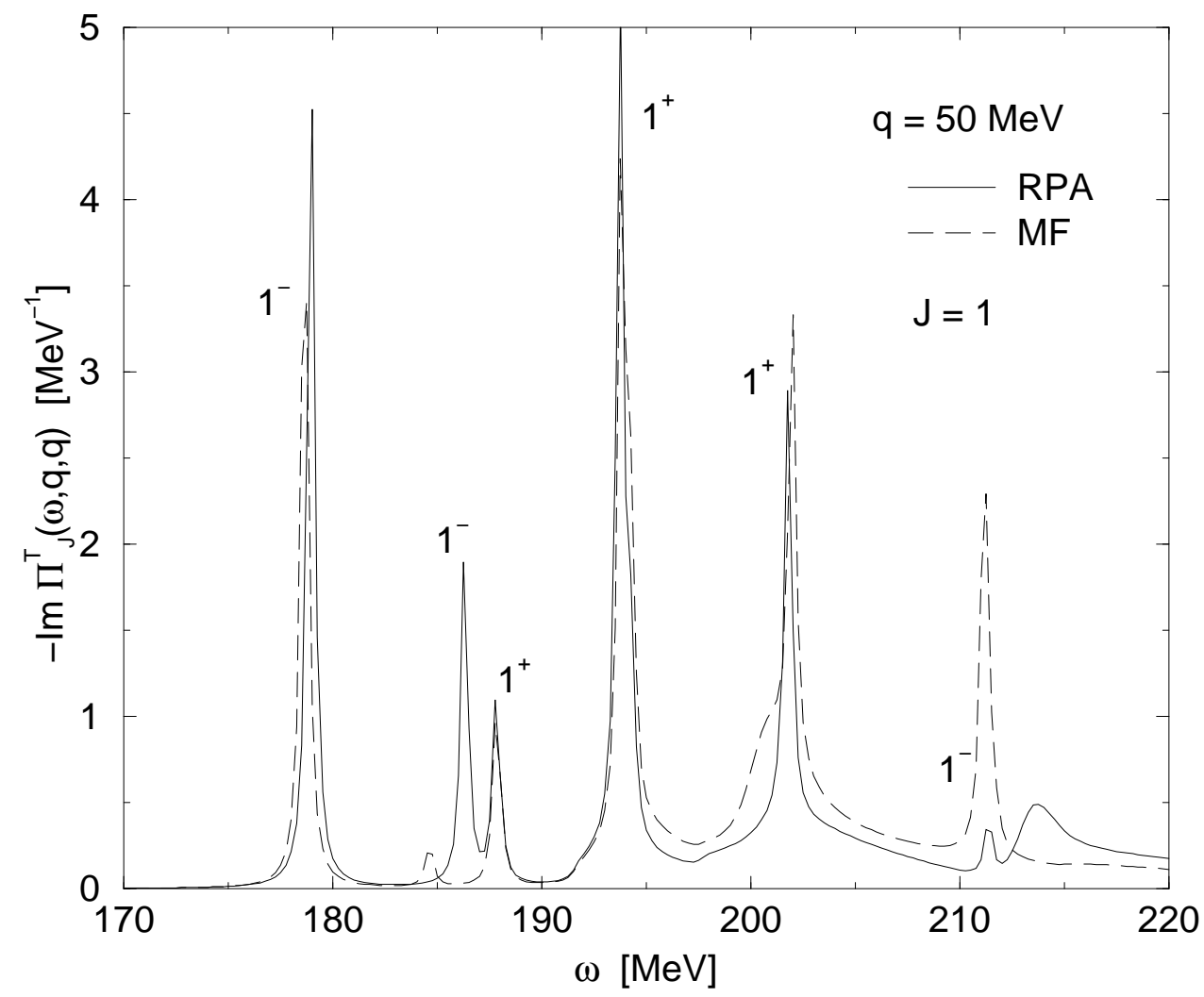

FIG. 3. Mean field and RPA result for the $J=1$ multipole of the transverse $K^{*}$ response in oxygen. 


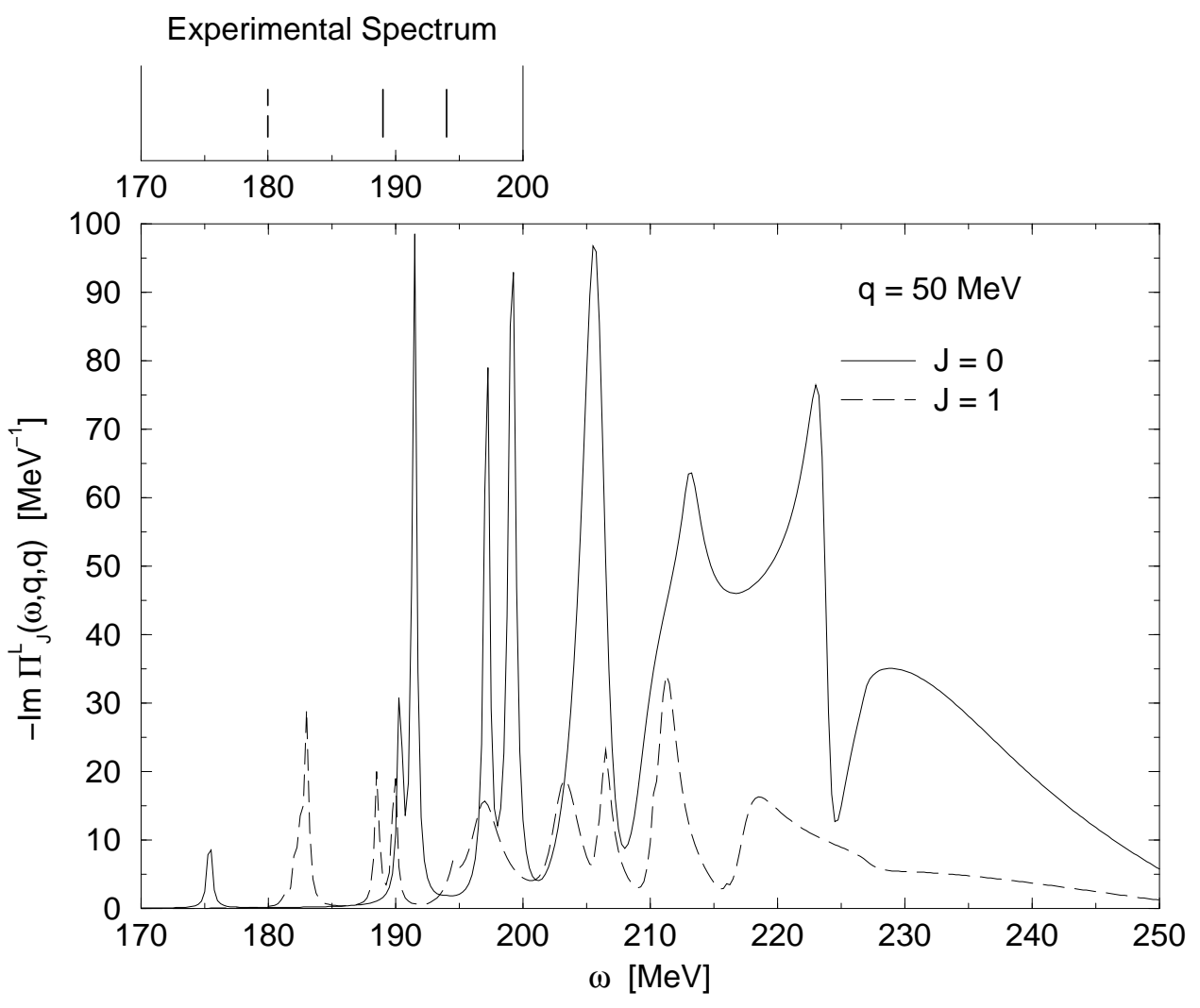

FIG. 4. Lowest order multipoles of the longitudinal $K^{*}$ response in calcium. 


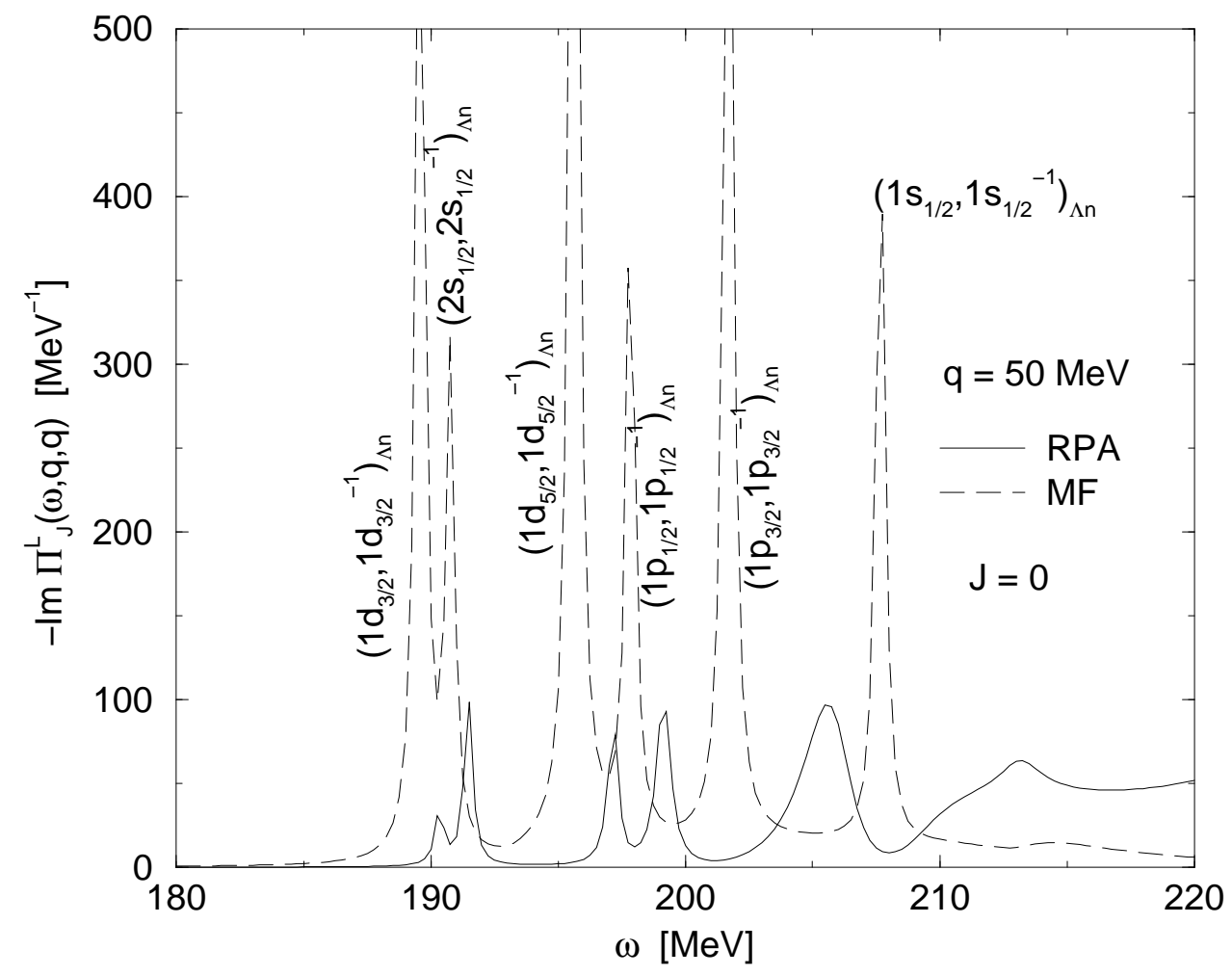

FIG. 5. Mean field and RPA result for the $J=0$ multipole of the longitudinal $K^{*}$ response in calcium. 


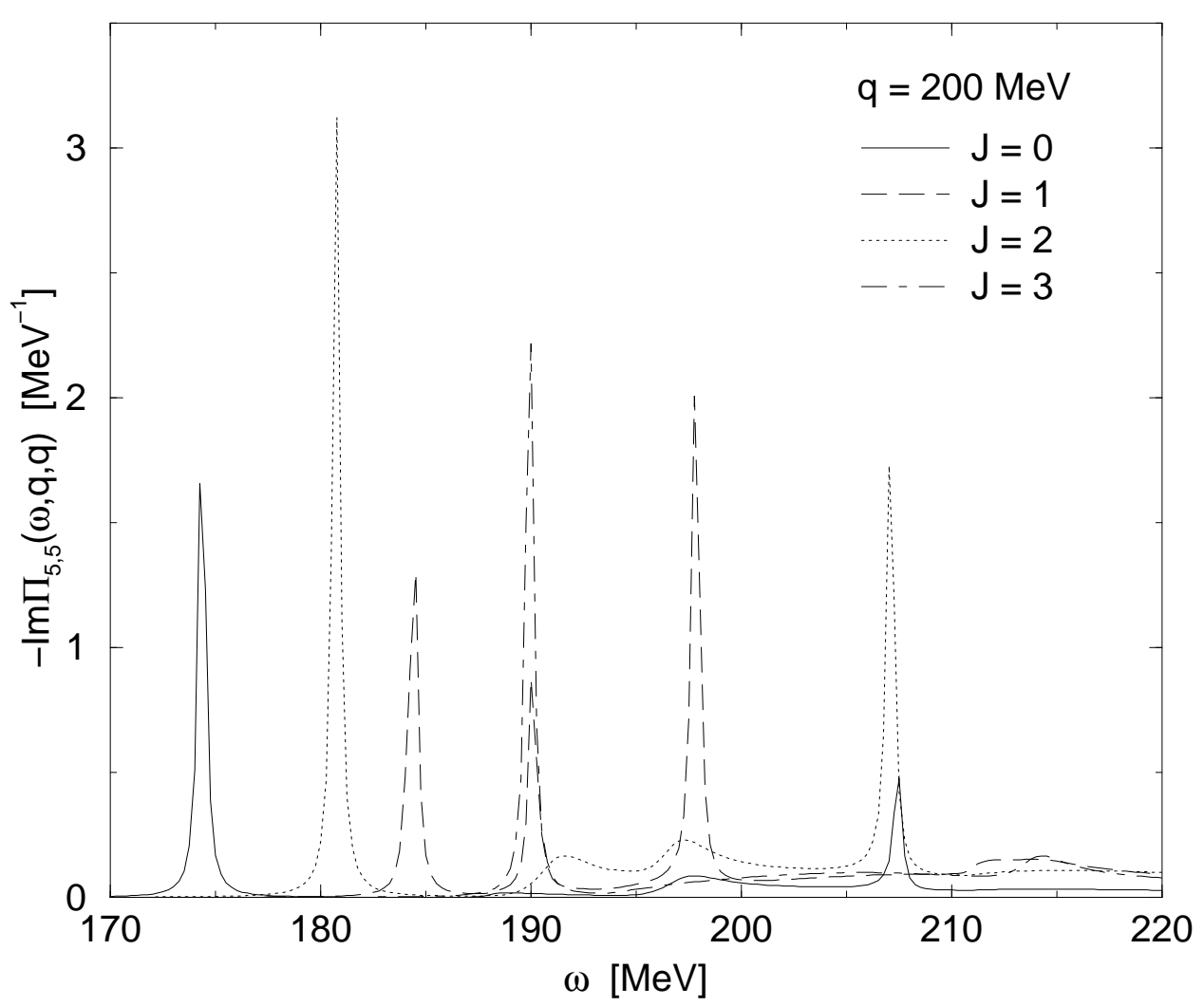

FIG. 6. Lowest order multipoles of the kaon response in ${ }^{16} \mathrm{O} \rightarrow{ }_{\Lambda}^{16} \mathrm{~N}$. 


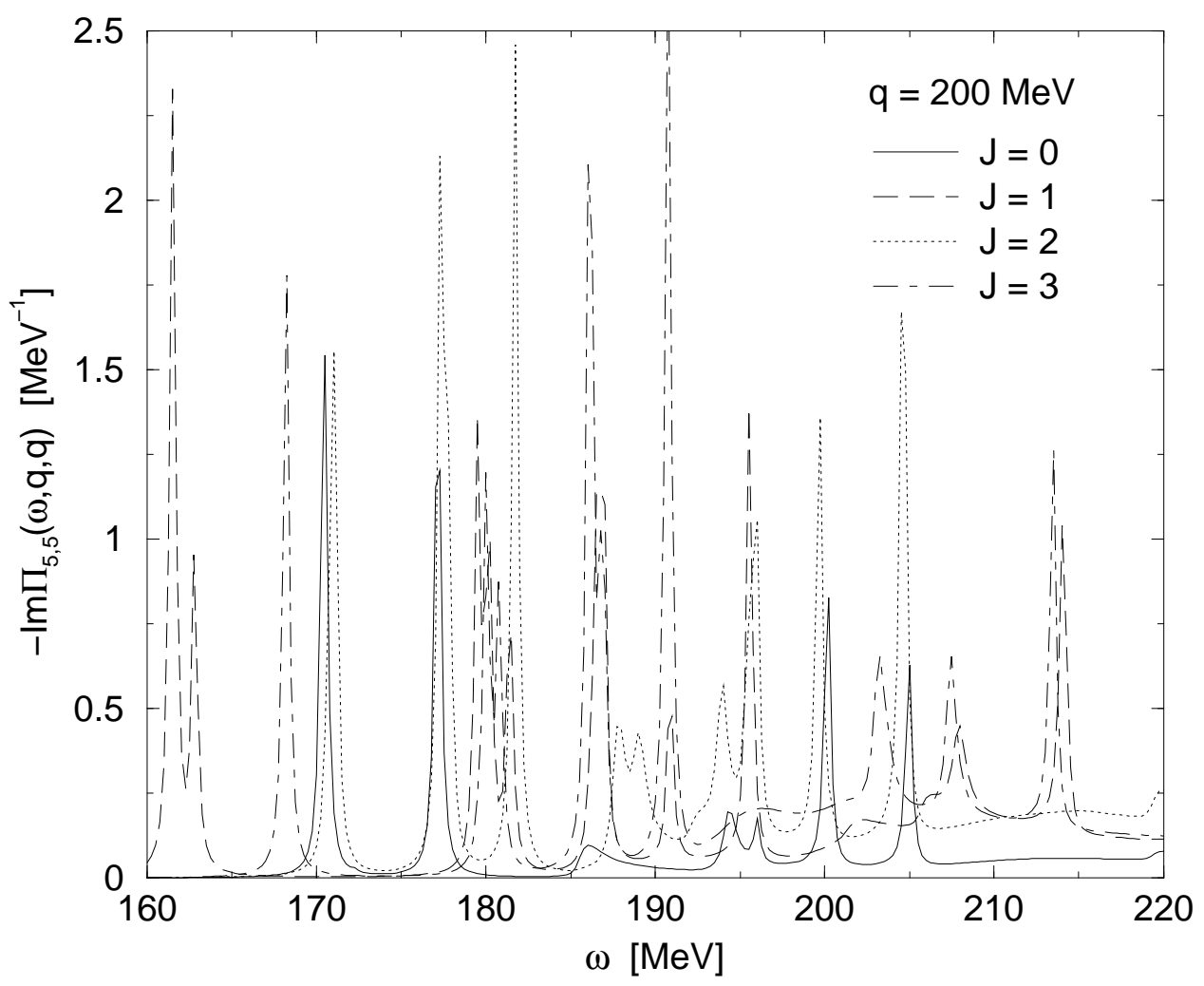

FIG. 7. Lowest order multipoles of the kaon response in ${ }^{40} \mathrm{Ca} \rightarrow{ }_{\Lambda}^{40} \mathrm{~K}$. 


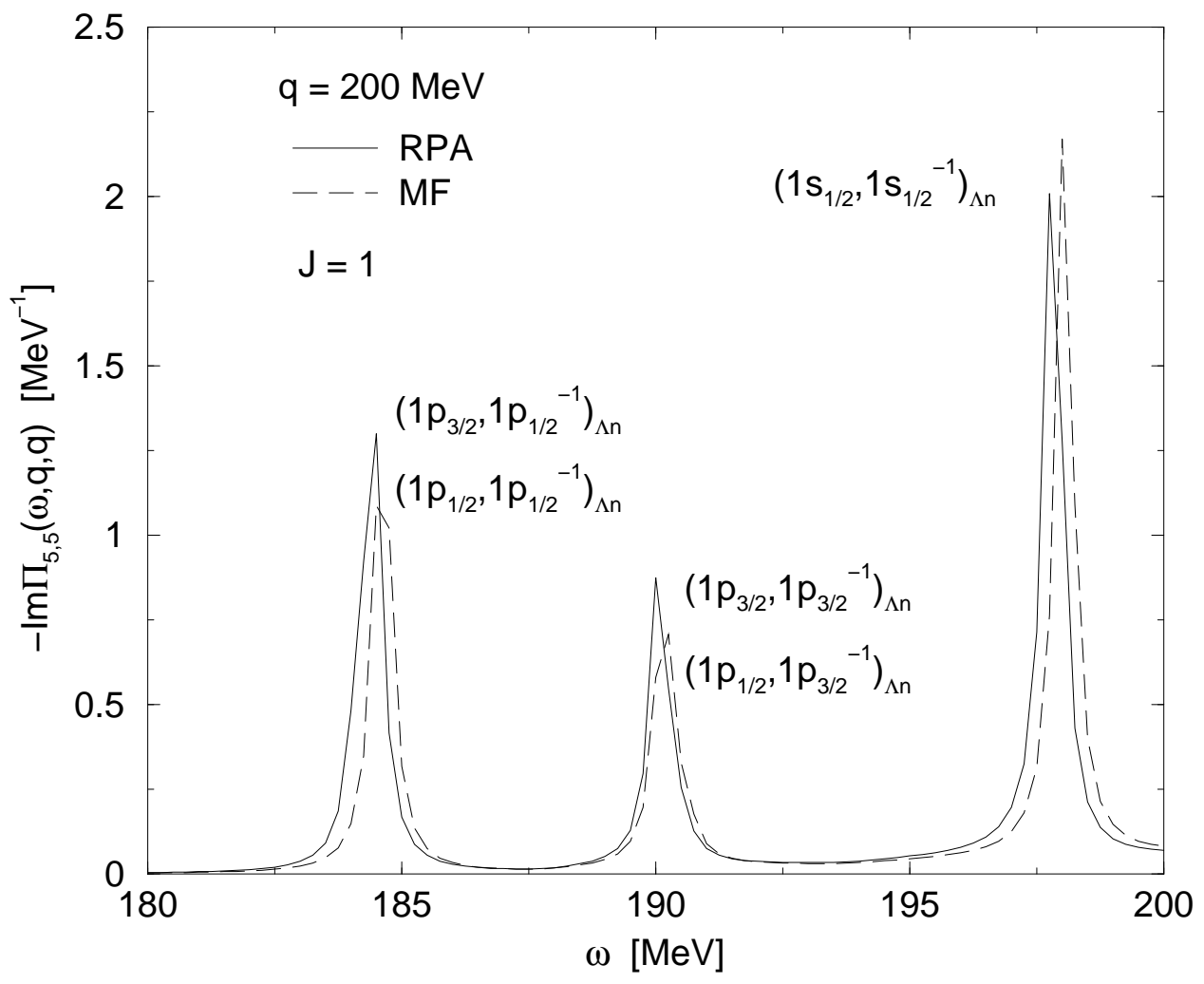

FIG. 8. Mean field and RPA result for the $J=1$ multipole of the kaon response in ${ }^{16} \mathrm{O} \rightarrow{ }_{\Lambda}^{16} \mathrm{~N}$. 